\title{
Poverty Estimates for New Hampshire Counties
}

\author{
Andrew Schaefer, Jessica A. Carson, and Marybeth J. Mattingly
}

$\mathrm{O}$ n October 20, 2017, the U.S. Census Bureau made available estimates of poverty and other indicators for 2016 for small geographic areas. In considering these data from the American Community Survey (ACS), it is important to pay close attention to the margins of error (MOE) before reaching any conclusions-especially when doing comparisons such as comparing poverty rates between counties and years.

The margins of error are quite high for small counties, making those estimates particularly unreliable and imprecise. For example, the recently released data show Coös County with a poverty rate of 11.7 percent, but the associated margin of error of 4.0 percentage points suggests a wide range of probable values (estimated poverty rate between of 7.7 and 15.7 percent). The estimates for child poverty are even more imprecise, due to even larger margins of error with this smaller population. As New Hampshire has small counties generally, county differences in these estimated poverty rates within New Hampshire are not substantially or statistically meaningful.

It is possible to do such comparisons but only by combining multiple years of ACS data to increase the statistical validity of the estimates. In our work, we rely on multi-year data to specifically provide more reliable estimates of poverty for New Hampshire counties. The table below uses the most recent five-year estimates from the ACS (2011-2015). These will be updated in December when the U.S. Census Bureau releases 2012-2016 data.

\section{ESTIMATED NUMBER AND PERCENT POOR FOR TOTAL AND CHILD POPULATION, BY NEW HAMPSHIRE COUNTY}

\begin{tabular}{lcccc|cccc|} 
& \multicolumn{3}{c|}{ TOTAL POPULATION } & \multicolumn{4}{c|}{ CHILDREN } \\
& Estimate & MOE & Percent & MOE & Estimate & MOE & Percent & MOE \\
\hline Belknap County, New Hampshire & 6,214 & 948 & $10.5 \%$ & $1.6 \%$ & 2,119 & 511 & $18.2 \%$ & $4.2 \%$ \\
\hline Carroll County, New Hampshire & 4,484 & 771 & $9.5 \%$ & $1.6 \%$ & 900 & 286 & $11.2 \%$ & $3.5 \%$ \\
Cheshire County, New Hampshire & 8,467 & 1,278 & $11.8 \%$ & $1.8 \%$ & 2,374 & 556 & $16.9 \%$ & $3.8 \%$ \\
\hline Coos County, New Hampshire & 4,257 & 487 & $14.1 \%$ & $1.6 \%$ & 1,202 & 209 & $21.7 \%$ & $3.5 \%$ \\
\hline Grafton County, New Hampshire & 9,609 & 1,182 & $11.6 \%$ & $1.4 \%$ & 2,372 & 516 & $15.5 \%$ & $3.3 \%$ \\
\hline Hillsborough County, New Hampshire & 34,681 & 2,533 & $8.8 \%$ & $0.6 \%$ & 10,367 & 1,078 & $11.7 \%$ & $1.2 \%$ \\
\hline Merrimack County, New Hampshire & 12,840 & 1,161 & $9.1 \%$ & $0.8 \%$ & 3,619 & 556 & $12.3 \%$ & $1.9 \%$ \\
\hline Rockingham County, New Hampshire & 16,192 & 1,561 & $5.5 \%$ & $0.5 \%$ & 4,586 & 709 & $7.3 \%$ & $1.1 \%$ \\
\hline Strafford County, New Hampshire & 12,997 & 1,303 & $11.1 \%$ & $1.1 \%$ & 3,118 & 528 & $12.9 \%$ & $2.1 \%$ \\
\hline Sullivan County, New Hampshire & 4,099 & 851 & $9.7 \%$ & $2.0 \%$ & 1,062 & 365 & $12.7 \%$ & $4.3 \%$ \\
\hline
\end{tabular}

Note: MOEs represent $95 \%$ confidence interval

Source: American Community Survey 2015 5-year Estimates, Table B17020

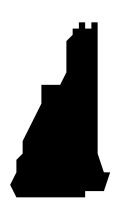

It is important to pay close attention to the margins of error (MOE) before reaching any conclusions-especially when doing comparisons such as comparing poverty rates between counties and years.

See related publications at carsey.unh.edu

- 2016 Child Poverty Rate Sees Largest Decline Since Before Great Recession (September 2017)

- Gains in Reducing Child Poverty, but Racial-Ethnic Disparities Persist (April 2017)

- Overall Declines in Child Poverty Mask Relatively Stable Rates Across States (September 2016)

Partial funding for this analysis provided by Wellspring Philanthropic Fund. 